the "Progress of Ophthalmology" (a subject with a frosted disk. The value of a lens must of course which you are all familar) simply mention to you depend upon its angle of refraction, whether it be a what I believe to be the most attractive feature of prism, cylinder,sphere or a combination. If we adopt our sessions, namely: the discussion which follows the method of gradation by the angle of refraction, the reading of papers. Dispense with this, or devote we shall have no more such confusion, as the student too short a time to it and our meetings are unprofitable. Cultivate and foster it, then the converse obtains.

Of course, it is highly essential, that we have a sufficient number of ably written papers for our consideration, but if too many are read, the object of our meeting together is defeated and we go hence almost as barren of results as if we had remained away. It is on this rock that the chairman and secretary are liable to founder; because in their efforts to provide material, they usually have to invite many to contribute; could they know before hand just who would respond favorably, but few mistakes could happen, but dealing with unknown numbers they are just as likely to have too many as too few, when disaster follows.

Would it not be wise to give the matter over to the executive committee, whose duty it should be to decide as to the papers which should be read before the Section, and those by title only?

Subjects are often presented which have cost their authors months of painstaking research and yet, though they be the most ably prepared, if no discussion follows, they might as well have been placed in some medical journal and sent to us for perusal in our homes, at our leisure. But if on the contrary a full and free discussion follows, we go away amply repaid for any sacrifice we have made; we are refreshed and greatly benefited by this comparing of ideas and experiences, lifted as it were out of the ruts of routine work to the higher plane of progressive Ophthalmology.

So in the place of an address by your chairman we will immediately proceed to the work which is before the Section.

\title{
GRADATION OF LENSES.
}

Read in the Section of Ophthalmology at the Forty-third Annual meetin of the American Medical Association, held at Detroit, Mich., June. 1892 .

BY DUDLEY S. REYNOLDS, A.M., M.D.,

PROFESSOR OF OPHTHALMOLOGY, OTOLOGY AND MEDICAL JURISPRUDENC IN THE HOSPITAL COLLEGE OF MEDICINE, MEDICAL DEPARTMENT OF THE CENTRAL UNIVERSITY OF KENTUCKY; SURGEON TO THE EYE AND EAR DEPARTMENT OF THE LOUISVILLE CITY IOSPITAL.

The gradation of lenses has been a perpetual source of discontent amongst all those who seek scientific accuracy, and the want of a uniform system is universally recognized. I had the honor, at the Ninth International Medical Congress, to read a brief paper on the necessity for reform in the manner of designating lenses, and in conclusion, I suggested the propriety of designating them according to the angle of refraction, as for example: begin with a lens the refracting powers of which equal an angle of $15^{\prime}$; the next in the series $30^{\prime} ; 1^{\circ} ; 1^{\circ} 30^{\prime} ; 2^{\circ} ; 2^{\circ} 30^{\prime}$ etc., up to the maximum angle of deviation of the pencil of refracted light. At the Cincinnati meeting of the American Medical Association, in May 1888, I presented the subject in a brief review of the principles, upon which lenses are constructed, and presented a table embracing a series of forty-two lenses, graded in minutes and degrees, according to the angle of refraction as determined by the angle of deviation the lens produced in a point of light transmitted through

must encounter in his attempts to determine the retem, or those denominated in fractions of the radius of the quadrant.

Professor Loring says, in discussing the subject: "The metre is taken as the unit, and each metre is called a dioptric. As the French metre is equal to thirty-seven inches, $1 \mathrm{D} .=\frac{1}{3} \mathrm{~T}$ expressed in the old style." Dr. Claibourne of New York, suggests that, " the dioptre is equivalent to $\frac{1}{3} \frac{1}{6}$ or $\frac{1}{40}$ according as the French or English inch is taken as the standard." Professor Landolt says: "One dioptre is to be taken as 37 Paris inches, or 39-5 English inches."

In England, distances are measured,as in the United States, by lines, inches, feet and yards, linear measure. In France, and some other countries, which have adopted the French system of millimeters, centimeters and meters, the metrical system of grading lenses harmonizes with this method of linear measurement, but it does not harmonize with either the English or American methods of linear measurement. To show how far this confusion extends, I invite your attention to the following, from Professor Noyes recent text book on diseases of the eye (p.91): "I have noted with the same optical error uncorrected, say $1 \mathrm{D}$. or $2 \mathrm{D}$., differences of vision varying between $\frac{20}{40}$ and $\frac{20}{10} \%$. All through the literature of this subject, are found just such confusing statements. Now, the state of vision, according to Professor Snellen's method,is determined by ascertaining the capacity of the eye to perceive objects under a definite angle, illuminated by parallel light. Assuming that the range of accommodation does not extend quite so far as twenty feet, this is taken as the proper distance, beyond the range of accommodation, where objects may be placed for the determination of the refraction of the eye, and the relative acuity of vision with parallel light. Twenty, therefore, meaning English feet, is the numerator, and the size of the test object for determining the angle of vision, the denominator of the fraction used to express the state of vision, uninfluenced by the accommodation.

The fractional system of dividing the quadrant of the crown glass sphere has become part of the classical literature of mathematical science. It was used for designating lenses for spectacles, beginning with Friar Bacon, about 1282, descending in unbroken lines, until 1867, when the International Ophthalmological Congress, held at Paris, appointed a committee to investigate and report in favor of a uniform system of designating lenses. The Committee reported in favor of the metrical scale of focal lengths, having an interval equal to a lense of two hundred and forty centimeters focus. Since that time, Pœtz and Flohr of Berlin, with their lenses graded in Prussian inches of focal lengths, and Nachet and Son, with their series, graded in Paris inches, have no longer just grounds of dispute with English opticians, for they too, have adopted the metrical scale of grading lenses, still measuring distances by the old English standard, and recording results by the haphazard methods of unreasoning custom.

Dr. Edward Jackson of Philadelphia, deserves the thanks of the scientific world for his successful work 
in advocacy of the adoption of the angle of deviation of refracted light as a test of the value of prisms. Following this line precisely in the footsteps of Dr. Jackson, we shall be forced to adopt the angle of refraction as the test of value of all lenses, and we should designate them accordingly, in minutes, and degrees, thus harmonizing the practical application of the science of optics with those principles employed in the determination of the optical properties of the human eye, with the same mathematical principles elucidated by the great Astronomers and Mathematicians of the world.

Now suppose we reverse the order of calculating the radius of curvature by dividing the quadrant of the sphere, and substitute the point of positive infinity as the beginning of our scale of gradation of refracting lenses?

To begin this way, we avoid the inharmonious arrangement of taking a medium refracting power, one meter in focal length, as the unit, and we likewise avoid the difficulty of securing a uniform medium as an index of refraction for the construction of our test lenses, and those we prescribe for our patients.

It is a fact, that we do require of the optician, a Iens having equal angular refraction with our test lens, and we do not require a specified medium out of which to construct the lens prescribed for the patient; it follows, therefore, that we reject the lens measured by focal length merely, as determined by any fixed standard of radius in the grinding tools, and require the crucial test of refracting power. Then, let us so determine our scale as to begin with parallel light, and denominate the refracting media according to their power to deviate that light in angular terms of minutes and degrees. The greatest confusion results from the common habit of writers and practitioners using the denominator of the fraction of the radius as synonymous with so many inches of focal length. It is just as common to observe practitioners using some of the cheap imitations of Snellen's test types, not one of which have either corresponding size or form of the original. I have measured the letters in a large number of socalled reprints and found none of them even approximately like Snellen's. Tests of acuity of perception and angle of refraction in the eye must always correspond, but this can never be possible without correctly graded test lenses, correctly graded test objects, and apportionment of distance in terms harmonious with the mensuration employed in grading the angular distances of test objects, and refraction of the test lenses. Neglect of these essentials impairs the value of nearly all the published statistical tables of clinical observations on all the varying forms of ametropia.

WHAT MAY BE CONSIDERED NORMAL CORNEAL ASTIGMATISM ? FROM KERATOMETRIC MEASUREMENTS OF THREE HUNDRED EYES.

Read in the Section of Ophthalmology at the Forty-third annual meeting of the American Iredical Association, held at Detroit, Mich., June, 1892.

\section{BY H. V. WÜRDEMANN, M.D.,} OF MILWAUKEE, WIS.

Keratometry is now considered an essential to the examination of the ocular refraction. As the principal seat of astigmatism is in the cornea there can be no doubt but that the measurement of its curvature is both time and labor saving; and in the near future this fact will be appreciated by every reputable ophthalmist. Correction of refractive errors is the most scientific portion of an oculist's work and cannot be done properly without several means of objective examination.

The ophthalmometer of Javal and Schiötz is conceded to be the most practical instrument for the estimation of the corneal curvature. In the accompanying instructions, ${ }^{17}$ in treatises and text books, the corneal is taken as equal to the whole astigmatic error. In 1882 Javal reported ${ }^{11}$ that in the measurement of 100 eyes in 96 per cent. the corneal corresponded exactly with the total astigmatism. His associate Nordensen ${ }^{16}$ followed later with a report of several hundred more, giving the same average. Since that time this statement has been accepted by most writers.

In 1889 Swan Burnett said ${ }^{3}$ that his experience with Javal's ophthalmometer tended to substantiate this although his percentage of difference between the total and the corneal astigmatism was greater. A few months ago the same author wrote ${ }^{4}$ : "It is a fact that we seldom find a cornea without a measurable degree of astigmatism." Normal corneal astigmatism he would place at .25 or $.50 \mathrm{D}$. according to the rule. G. Lindsay Johnson says ${ }^{13}$ that the majority of persons possess a corneal astigmatism of less than $.75 \mathrm{D}$. Bull finds ${ }^{2}$ an astigmatism of from .25 to $.75 \mathrm{D}$. with the rule in 80 per cent. of his cases. Doubtless many others* have noticed the disparity between the corneal and total measurements but have not published their observations. However this may be it seems marvelous that the statement of Javal has gone so long unchallenged.

The usual directions for keratometry with the ophthalmometer are: After finding the direction of the principal meridians to carry the movable plaque toward the stationary until the images touch and to judge of the degree of the astigmatism, after turning to the meridian of less refraction, by the number of overlapping steps. ${ }^{17}{ }^{3}$ This method is open to the objection of inaccuracy in that the personal equation of each observer admits of an error which may amount to .50 or $1.00 \mathrm{D}$. It will do very well for rough work but closer results may be obtained, by first approximating the plaques and finding the direction of the astigmatism, then moving the stepped plaque until the images of the first two black interspaces are equal. It is objected by Weiland ${ }^{21}$ that the refracting quartz prism of the telescope is not achromatic, so that the edges of the images are not sharply defined, making it impossible to get accurate contact. One can more accurately estimate the size of two black objects than judge when two white surfaces exactly touch. Of two equal squares, the one white on a black back-ground, the other black on a white back-ground, when contrasted the former appear larger. The edges of white objects when approximated appear to merge into each other

* After this article was written I came across the following by a writer in an English ophthalmic journal. He published the astigmatism found by the ophthalmometer in 139 eyes, and in a paraliel colum the cylndrical lenses hinally agreed upon as the proper ones, after comparison of results obtained thalmoscope and retinoscope. In 62 eyes he claims that the ophthal mometer indicated exactly the true amount of astigmatism. In 14 the discrepancy was but $25 \mathrm{D}$., and in 26 it was a whole diopter or over, the greatest difference being $3 \mathrm{D}$. He says that in many cases in which the in the Ophthalmic Review, vol. $x$, No.117. 